Acta Psychologica 30 Attention and Perfurmance II (W. G. Koster, ed.) 1969, 54-57

(C) North-Holland Oublishing Company, Amsterdan

\title{
SEQUENTIAL DEPENDENCIES AS A DETERMINANT OF CHOICE REACTION TIME: A SUMMARY 1
}

\author{
SYLVAN KORNBLUM 2 \\ Mental Healih Research Institute, University of Michigan, \\ Ann Arbor, Mich., U.S.A.
}

There are at least three different ways of varying the average stimulus information $(H)$ in a sequence: (a) where the stimuli, or alternatives, occur equiprobably and independently, one can vary the number $(K)$ of different stimuli that are used; (b) where the number of alternatives is fixed, one can vary the absolute probabilities of occurrence of the stimuli, still allowing them to occur independently; finally, (c) where the number ' $f$ alternatives is fixed, and the stimuli oscur equiprobably, onestep sequential dependencies [i.e., $p(i \mid j) \neq p(i)$ ] may be introduced in the construction of the sequences. In order to simplify the discussion, the latter cale will be restricted to instances in which $p(i \mid j)-p(h \mid k)$ (where $i \neq j$, and $h \neq k$ ). It can be shown that $H$ and the conditional probability of non repetition [i.e., $p(i \mid j, i \neq j)$, henceforth referred to by ' $p$ '] are usually confounded in (b) and necessarily confounded in all instances of (a), and (c) where $0 \leqslant p \leqslant 1 / K$. Hence, inferences based on experimental evidence from which this source of confounding has not been removed, and which imply that the increase in choice reaction time (RT) can be accounted for by the increase in $H$, are tenuous at best.

One interesting property of varying $H$ by means of procedure (c) above, is that within a given range of $H$ and $p$, there exist pairs of different values of $p$ which will generate pairs of sequences with identical values of $H$ within pairs. In principle, therefore, $H$ and $p$ may be experimentally unconfounded by comparing the overall mean RT for sequences within such equi-information pairs, where one sequence has a higher $p$ value than the other. This is precisely the property which was exploitis in the construction of the stimulus sequences for the serial

1 This is a brief summary of the talk which was presented at the Donders Centenary Symposium. The material is based on a paper entitled: 'Sequential determinants of information processing in :erial and discrete ciloice reaction time,' Psychol. Rev., 1969, in press.

2 I thanls the University of Michigen whose sole support has made this research possible. 
and the discrete experiments to be reported. Both experiments consisted of a four-choice kevboard task. In the serial experiment, the stimuli were four neon lights and the time between a response and the presentation of the next stimulus (R-S interval) was $140 \mathrm{msec}$; in the discrete experiment, the stimuli were the digits one through four, and the R-S interval was approximately three seconds. Eight different stimulus sequences with equiprobable stimuli were used in each experiment. There were six sequences that formed three equi-information pairs such that for one sequence in the pair (low-p) $p<1 / K$, and for the other sequence in the pair (high-p) $p>1 / K$; there was also a two-bit sequence which was unique with $p=1 / K$ and finally there was one unpaired high-p sequence.

The least that the Information Hypothesis would lead one to expect is that with all other things being equal, a one-to-one correspondence would be obtained between transmitted information and the overall mean RT. The results for the equi-information pairs within each experiment indicated that the overall mean RT for the high-p sequences was significantly longer than for the low- $p$ sequences. Furthermore, the slope of the overall mean RT with respect to $H$ for the set of high-p sequences was significantly less than for the set of low-p sequences. The overall error rate was approximately constant across the eight sequences. It was, therefore, concluded that $\boldsymbol{H}$ was neither necessary nor sufficient to account for ine data.

After partitioning the data into repetitions and non-repetitions, it was found that the mean RTs for repetitions and non-repetitions were decreasing linear functions (having no statistically significant non-linear component) of their respective conditional probabilities, with equal slopes in each experiment. The overall mean RT can, therefore, be expressed as a parabolic function of $p$ :

$\overline{\mathrm{RT}}=p^{2}\left[-m_{k},\left(K^{2}-K\right)\right]+p\left[(K-1)\left(b_{n r, k}-b_{r, k}+2 m_{k}\right)\right]-m_{k}+b_{r}, k$

where:

$\overline{\mathbf{R T}}=$ the overall mean reaction time

$p=$ the conditional probability of non-repetition $p(i \mid j, i \neq j)$, i.e., the probability that stimulus (i) was presented on trial $n$, given that a different stimulus was presented on trial $(n-1)$

$m=$ the slope with respect to $p(i \mid j)$ of the RT for repetitions and non-repetitions 


$$
\begin{aligned}
& b_{r}=\text { the inte cept for repetitions } \\
& b_{n r}=\text { the intercept for non-repetitions } \\
& k, K=\text { the number of alternatives. }
\end{aligned}
$$

It is easy to calculate those values of $p$ which are required to generate secquences with $H$ values of two and three bits with eight alternatives, and one and two bits with four alternatives. When these $p$ velues are substituted in equation 1 , and if it is assumed that the overall n.ean RT is approximated by a linear function within a one bit range, then an estimate of the slope of the overall mean RT with respect to $H$ can be calculated for the four-choice, and the eight-choice case. These slopes are:

$$
\begin{aligned}
& \text { for eight-choice: } 0.286 m_{k}+0.50 \Delta b_{k} \\
& \text { for four-choice: } 0.403 m_{k}+0.55 \Delta b_{k}
\end{aligned}
$$

where:

$\Delta b_{k}=b_{n, j k}-b_{r, k}$ (i.e., the difference in the intercept for repetitions and non-repetitions, with $k$ number of alternatives).

There is good evidence to indicate that $\Delta b$ is an increasing functiun of $K$, even though it is difficult to ascertain what effects, if any, $K$ may have on $m$. As long as this increase in $\Delta b$ exceeds a minimum value, it is clear from equations 2 that the slope of the overall mean RT with respect to $H$ will increase with the number of alternatives. This conclusion is verified by an examination of Hyman's own two, four, and eight choice data (HYMAN, 1953), Thus the high correlations that have been reported between $H$ and the overall mean RTs for conditions with different numbers of alternatives, appear to a fortuituous consequence of $\Delta b$ being an increasing function of $K$, jointly with the highly restricted range in which $\boldsymbol{H}$ was allowed to vary for each value of $K$.

Finally, it can be shown that the slope of the overall mean RT with respect to $H$ will increase either as a result of decreasing the S-R compatibility of the rask, or as a result of increasing the number of alternatives; when these two variables are varied jointly, their effect is multiplicative, i.e., they interact as a consequence of the eff 1 ct that they each have on $m$ and $\Delta b$.

\section{REFEFENCE}

HyMan, R., 1953. Stimulus information as a determinant of reaction time. J. exp. Psychol. 45, 188-196. 
Discussion

Falmagne: My question is related to the range of ine values of the probability of repetitions. There is a part of the range you have not been considering. What is going to happen if the probability of a repetition is high, for instance $99 \%$ ? By extrapolation of the regression lines you should be able to predict what is the value of something which is close to either a simple reaction time or a Donders c-reaction time.

Kornblum: If you look at the figure in which the overall mean reaction times are plotted as a function of the conditional probability for non-repetitions, you can see that for a conditional probability of non-repetitions equals zero, i.e. that the probability of repetitions is one (a simple reaction time task), the intercept is $195 \mathrm{msec}$ in a serial task, which corresponds closely to a simple visual reaction time. It is about $300 \mathrm{msec}$ in the discrete experiment, which is a bit high, but the task is less compatible. 